Pacific Journal of Mathematics

THE REMAINDER TERMS ASPECT OF THE THEORY OF TH 


\title{
THE REMAINDER TERMS ASPECT OF THE THEORY OF THE RIEMANN ZETA-FUNCTION
}

\section{Ka-Lam KueH}

\begin{abstract}
Assuming the Riemann hypothesis for Riemann zeta-function $\zeta(s)$, let $R(u)$ and $S(t)$ denote the remainder terms for the prime number theorem (suitably normalized) and the zero counting formula for $\zeta(s)$ respectively. We analyze the relation between $R(u)$ and $S(t)$, which generalizes A. Guinand's work.
\end{abstract}

0. Introduction. In this paper, the general form of transform $T$ on $L^{2}(0, \infty)$ defined by a kernel $\varphi$ is given by

$$
T f(t)=\lim _{U \rightarrow \infty} \int_{0}^{U} f(u) \varphi(t u) d u \quad \text { in } L^{2}(0, \infty)
$$

for $f \in L^{2}(0, \infty)$. Throughout this paper, we assume the Riemann hypothesis for the Riemann zeta-function $\zeta(s)$. It is concerned with the remainder terms $R(u)$ in the prime number theorem (suitably normalized) and $S(t)$ in the zero counting formula for the Riemann zeta-function; see (2.1) and (2.4). Gallagher's version [2, Theorem 1] of the Guinand's summation formula, $(0.0)$ below, gives a symmetric relation between $R(u)$ and $S(t)$ :

$$
\int_{0}^{\infty} f(u) d R(u)=\int_{0}^{\infty} \hat{f}(t) d S(t)
$$

where $\hat{f}(t)=\int_{0}^{\infty} f(u) \cos t u d u$, for a suitable class of functions $f(u)$ with "good" growth condition on $f(u)$. In particular, it is possible to take as $f(\cdot)$ in $(0.0)$ the characteristic function of the interval $[0, u]$, and on taking integration by parts, we get

$$
\sqrt{\frac{2}{\pi}} \frac{R(u)}{u}=-\int_{0}^{\infty} \frac{S^{*}(t)}{t} k(t u) d t
$$

where

$$
S^{*}(t)=S(t)-S\left(0^{+}\right) \quad \text { and } \quad k(\theta)=\sqrt{\frac{2}{\pi}} \theta \frac{d}{d \theta} \frac{\sin \theta}{\theta} .
$$


A. Guinand [3] observed that $k(\theta)$ is the kernel of a Hankel transform of order $3 / 2$, which is an involution on $L^{2}(0, \infty)$. So we have

$$
-\frac{S^{*}(t)}{t}=\sqrt{\frac{2}{\pi}} \int_{0}^{\infty} \frac{R(u)}{u} k(t u) d u
$$

since $S(t)=O(\log t / \log \log t)$ due to Littlewood [6], and hence $S^{*}(t) / t$ $\in L^{2}(0, \infty)$.

Equations (0.1) and (0.2) suggest a symmetric relation between $R(u)$ and $S(t)$. For a function $f(x)$, the Riemann-Liouville fractional integral is defined by

$$
I_{\alpha} f(x)=f_{\alpha}(x)= \begin{cases}\frac{1}{\Gamma(\alpha)} \int_{0}^{x}(x-y)^{\alpha-1} f(y) d y & (\alpha>0), \\ f(x) & (\alpha=0)\end{cases}
$$

and we have

$$
I_{\alpha+\beta} f(x)=I_{\alpha} I_{\beta} f(x)
$$

for $\alpha \geq 0, \beta \geq 0$. For $\alpha$ an integer $f_{\alpha}(x)$ turns out to be an iterated integral. As a generalization of Guinand's work, we consider the Riemann-Liouville fractional integral on $R(u)$ and $S(t)$, and construct the kernel

$$
K(\alpha, m, \theta)=\sqrt{\frac{2}{\pi}} \theta^{\alpha+1}\left(\frac{d}{d \theta}\right)^{m+1} \frac{\sin _{\alpha}(\theta)}{\theta^{\alpha+1}} \quad(\theta>0)
$$

where $m$ is an integer and $\alpha$ is real, and the equation similar to $(0.1)$

$$
\sqrt{\frac{2}{\pi}} \frac{R_{\alpha}(u)}{u^{m+1}}=(-1)^{m+1} \int_{0}^{\infty} \frac{S_{m}^{*}(t)}{t^{\alpha+1}} K(\alpha, m, t u) d t .
$$

Note that $S_{1}(t)=O\left(\log t /(\log \log t)^{2}\right)$ due to Littlewood [6], and hence

$$
S_{m}^{*}(t)=S_{m}(t)-\frac{1}{m !} S\left(0^{+}\right) t^{m}=O\left(t^{m}\right) \quad(t \rightarrow+\infty)
$$

for $m \geq 1$, and

$$
S_{m}^{*}(t)=O\left(t^{m+1}\right) \quad\left(t \rightarrow 0^{+}\right)
$$

for $m \geq 0$.

We give first in Theorem 1 the conditions on $\alpha, m$ for which equation (0.4) holds good, and then in Theorem 2 the conditions on $\alpha, m$ for which $K(\alpha, m, \theta)$ defines a bounded and invertible transform on $L^{2}(0, \infty)$. In particular, $K(m, m, \theta)$ for $m \geq-1$ is an involution 
on $L^{2}(0, \infty)$. Thus corresponding to $(0.2)$ we will be able to show in Theorem 3

$$
\frac{S_{\alpha}^{*}(t)}{t^{m+1}}=(-1)^{m+1} \sqrt{\frac{2}{\pi}} \int_{0}^{\infty} \frac{R_{m}(u)}{u^{\alpha+1}} K(\alpha, m, t u) d u
$$

for suitable $\alpha$ and $m$, and furthermore

$$
\int_{0}^{\infty}\left|\frac{S_{m}^{*}(t)}{t^{m+1}}\right|^{2} d t=\frac{2}{\pi} \int_{0}^{\infty}\left|\frac{R_{m}(u)}{u^{m+1}}\right|^{2} d u
$$

for $m=0,1,2,3, \ldots$.

1. Averaging operators. To pursue the object of this paper, we have to introduce the averaging operator.

For $f \in L^{2}(0, \infty)$, we define the transforms $A_{\alpha}$ and $B_{\beta}$ by

$$
\begin{array}{ll}
A_{\alpha} f(x)=x^{-\alpha} \int_{0}^{x} y^{\alpha-1} f(y) d y & \left(\alpha>\frac{1}{2}\right), \\
B_{\beta} f(x)=x^{-\beta} \int_{x}^{\infty} y^{\beta-1} f(y) d y & \left(\beta<\frac{1}{2}\right) .
\end{array}
$$

LEMMA 1.1. The $A_{\alpha}$ and $B_{\beta}$ are bounded operators on $L^{2}(0, \infty)$ and

$$
\left\|A_{\alpha}\right\|_{2} \leq\left(\alpha-\frac{1}{2}\right)^{-1}, \quad\left\|B_{\beta}\right\|_{2} \leq\left(\frac{1}{2}-\beta\right)^{-1} \text {. }
$$

Proof. By (9.9.8) and (9.9.9) of [4], the results follow immediately.

LEMMA 1.2. For all values of $\alpha$ and $\beta$ for which $A_{\alpha}$ and $B_{\beta}$ are defined, we have

$$
\begin{array}{ll}
A_{\alpha} B_{\beta}=\frac{B_{\beta}+A_{\alpha}}{\alpha-\beta}=B_{\beta} A_{\alpha}, \\
A_{\alpha} A_{\beta}=\frac{A_{\beta}-A_{\alpha}}{\alpha-\beta}=A_{\beta} A_{\alpha} \quad(\alpha \neq \beta), \\
B_{\alpha} B_{\beta}=\frac{B_{\beta}-B_{\alpha}}{\beta-\alpha}=B_{\beta} B_{\alpha} \quad(\alpha \neq \beta) .
\end{array}
$$

Proof. Note first that the unitary involution $J$, defined by

$$
J f(x)=\frac{1}{x} f\left(\frac{1}{x}\right),
$$

intertwines the operators $A_{\alpha}$ and $B_{1-\alpha}: J A_{\alpha}=B_{1-\alpha} J$.

It suffices to prove the first two equations on the left. The first equation on the right then follows by conjugation by $J$, the second 
equation on the right by symmetry, and the third pair of equations follows from the second pair by conjugation by $J$.

For continuous $f(x)$ with compact support in $(0, \infty)$, we have

$$
\begin{aligned}
A_{\alpha} B_{\beta} f(x)= & x^{-\alpha} \int_{0}^{x} y^{\alpha-1} y^{-\beta} \int_{y}^{\infty} z^{\beta-1} f(z) d z d y \\
= & x^{-\alpha}\left[\frac{y^{\alpha-\beta}}{\alpha-\beta} \int_{y}^{\infty} z^{\beta-1} f(z) d z\right]_{y=0}^{y=x} \\
& +x^{-\alpha} \int_{0}^{x} \frac{y^{\alpha-\beta}}{\alpha-\beta} y^{\beta-1} f(y) d y, \\
A_{\alpha} A_{\beta} f(x)= & x^{-\alpha} \int_{0}^{x} y^{\alpha-1} y^{-\beta} \int_{0}^{y} z^{\beta-1} f(z) d z d y \\
= & x^{-\alpha}\left[\frac{y^{\alpha-\beta}}{\alpha-\beta} \int_{0}^{y} z^{\beta-1} f(z) d z\right]_{y=0}^{y=x} \\
& -x^{-\alpha} \int_{0}^{x} \frac{y^{\alpha-\beta}}{\alpha-\beta} y^{\beta-1} f(y) d y
\end{aligned}
$$

from which the first two equations on the left follows. The general cases then follow by continuity of bounded operators.

LEMMA 1.3. The operator Id $-(2 \alpha-1) A_{\alpha}$ is unitary on $L^{2}(0, \infty)$ for each $\alpha>\frac{1}{2}$.

Proof. We show first

$$
A_{\alpha}^{*}=B_{1-\alpha} \quad \text { for } \alpha>1 / 2 \text {. }
$$

It suffices by continuity to show that

$$
\left(A_{\alpha} f, g\right)=\left(f, B_{1-\alpha} g\right)
$$

for continuous functions $f, g$ with compact support. The left is

$$
\begin{aligned}
\int_{0}^{\infty} x^{-\alpha} \int_{0}^{x} y^{\alpha-1} f(y) d y \bar{g}(x) d x \\
=-\int_{0}^{\infty} \int_{0}^{x} y^{\alpha-1} f(y) d \int_{x}^{\infty} z^{-\alpha} \bar{g}(z) d z .
\end{aligned}
$$

On integrating by parts, the integrated terms drop out, leaving

$$
\begin{aligned}
\int_{0}^{\infty} \int_{x}^{\infty} z^{-\alpha} \bar{g}(z) d z d \int_{0}^{x} y^{\alpha-1} f(y) d y \\
\quad=\int_{0}^{\infty} f(x) x^{\alpha-1} \int_{x}^{\infty} z^{-\alpha} \bar{g}(z) d z d x,
\end{aligned}
$$

which is the right side. 
Now for each real $\alpha>1 / 2$, we have by (1.1)

$$
\left(\mathrm{Id}-(2 \alpha-1) A_{\alpha}\right)^{*}=\mathrm{Id}-(2 \alpha-1) B_{1-\alpha}
$$

and by Lemma 1.2

$$
\begin{aligned}
& \left(\mathrm{Id}-(2 \alpha-1) B_{1-\alpha}\right)\left(\mathrm{Id}-(2 \alpha-1) A_{\alpha}\right) \\
& \quad=\mathrm{Id}-(2 \alpha-1) B_{1-\alpha}-(2 \alpha-1) A_{\alpha}+(2 \alpha-1)^{2} \frac{A_{\alpha}+B_{1-\alpha}}{2 \alpha-1} \\
& \quad=\mathrm{Id},
\end{aligned}
$$

which proves Lemma 1.3.

Let $h$ be a bounded measurable function on $(0, \infty)$. For each function $f \in L^{1}(0, \infty)$, we define

$$
(H f)(x)=\int_{0}^{\infty} f(y) h(x y) d y .
$$

Provided in addition $H$ is a bounded operator on $L^{1}(0, \infty) \cap$ $L^{2}(0, \infty)$, i.e.

$$
\|H f\|_{2} \ll\|f\|_{2} \quad \text { for } f \in L^{1}(0, \infty) \cap L^{2}(0, \infty)
$$

we may extend the operator $H$ by continuity to a bounded operator on all of $L^{2}(0, \infty)$. In particular, in this case

$$
(H f)(x)=\lim _{Y \rightarrow \infty} \int_{0}^{Y} f(y) h(x y) d y \quad \text { in } L^{2}(0, \infty)
$$

for each $f \in L^{2}(0, \infty)$.

LEMMA 1.4. Let $H$ be the bounded operator on $L^{2}(0, \infty)$ defined as above by a bounded measurable kernel $h$ satisfying (1.2). Then for each $\alpha>1 / 2$, we have

$$
A_{\alpha} H=H B_{1-\alpha} .
$$

Moreover, the operator (1.3) is defined as above by the kernel $A_{\alpha} h$.

Proof. We observe first that $A_{\alpha} h$ is a bounded measurable function. Next, for $f \in L^{1} \cap L^{2}$, we have

$$
\begin{aligned}
A_{\alpha} H f(x) & =x^{-\alpha} \int_{0}^{x} y^{\alpha-1} \int_{0}^{\infty} h(y z) f(z) d z d y \\
& =\int_{0}^{\infty}\left\{x^{-\alpha} \int_{0}^{x} y^{\alpha-1} h(y z) d y\right\} f(z) d z
\end{aligned}
$$


and

$$
\begin{aligned}
H B_{1-\alpha} f(x) & =\int_{0}^{\infty} h(x y) y^{\alpha-1} \int_{y}^{\infty} z^{-\alpha} f(z) d z d y \\
& =\int_{0}^{\infty}\left\{z^{-\alpha} \int_{0}^{z} y^{\alpha-1} h(x y) d y\right\} f(z) d z
\end{aligned}
$$

with both interchanges justified by Fubini's theorem. Each of the factors $\{\cdots\}$ reduces to $A_{\alpha} h(x z)=A_{\alpha} g(x)$ where $g(x)=h(x z)$.

Denoting by $H_{\alpha}$ the operator defined by $A_{\alpha} h$ on $L^{1} \cap L^{2}$, we thus have

$$
H_{\alpha} f=A_{\alpha} H f=H B_{1-\alpha} f
$$

for $f \in L^{1} \cap L^{2}$. For such $f$,

$$
\left\|H_{\alpha} f\right\|_{2}=\left\|A_{\alpha} H f\right\|_{2} \leq \frac{1}{\alpha-1 / 2}\|H f\|_{2} \ll\|f\|_{2},
$$

with an implicit constant depending on $\alpha$. It follows that $H_{\alpha}$ is a bounded operator on $L^{1} \cap L^{2}$ and thus $H_{\alpha}$ extends by continuity to a bounded operator on $L^{2}$. By continuity, the equation (1.4) now holds for $f \in L^{2}$, giving the assertions of Lemma 1.4 .

2. An explicit formula. It is known that $(s-1) \zeta(s)$ is an entire function and

$$
\frac{\zeta^{\prime}}{\zeta}(s)=-\sum_{n=1}^{\infty} \frac{\Lambda(n)}{n^{s}} \quad(\operatorname{Re} s>1)
$$

where $\Lambda(n)$ is the von Mangoldt function.

Put

$$
P(u)=-\frac{1}{2} \sum_{n \leq e^{u}}^{\prime} \Lambda(n) n^{-1 / 2}, \quad Q(u)=-2 \sinh \frac{1}{2} u
$$

the prime on the summation here and in the following means if $e^{u}$ is an integer, then the last term is weighted with $1 / 2$. A version of the prime number theorem states that $\sum_{n \leq x} \Lambda(n)=x+O\left(x^{1 / 2} \log ^{2} x\right)$ see Davenport [1].

Define

$$
R(u)=P(u)-Q(u)
$$


We have that $R(u)=O\left(u^{2}\right)$ which implies the prime number theorem with remainder term, and for $\alpha \geq 0$

$$
\begin{aligned}
R_{\alpha}(u)= & -\frac{1}{2} \frac{1}{\Gamma(\alpha+1)} \sum_{n \leq e^{u}}^{\prime} \Lambda(n) n^{-1 / 2}(u-\log n)^{\alpha} \\
& +\sinh _{\alpha}\left(\frac{1}{2} u\right) /\left(\frac{1}{2}\right)^{\alpha+1} ;
\end{aligned}
$$

note that $\left.I_{\alpha}(f)\right|_{c y}=\left.c^{\alpha} I_{\alpha}(g)\right|_{y}$ where $g(y)=f(c y)$.

Now define for $t>0$ not an ordinate of a zero of $\zeta(s)$

$$
N(t)=\#\left\{\frac{1}{2}+i \gamma \mid \zeta\left(\frac{1}{2}+i \gamma\right)=0,0<\gamma \leq t\right\},
$$

and $N(t)=\frac{1}{2}\left(N\left(t^{+}\right)+N\left(t^{-}\right)\right)$for $t$ the imaginary part of a zero of $\zeta(s)$. By the argument principle, we see that for $T>0$ not an ordinate of a zero of $\zeta(s)$

$$
N(T)=\frac{1}{2 \pi i} \int_{\Gamma_{T}} \frac{\zeta^{\prime}}{\zeta}(s) d s
$$

where $\Gamma_{T}$ is the line running from $\infty+i T$ to $\frac{1}{2}+i T$ to $\frac{1}{2}-i T$ to $\infty-i T$, and a Cauchy principal value is taken at each zero of $\zeta(s)$ on $\Gamma_{T}$.

In view of $\zeta(\bar{S})=\overline{\zeta(s)}$, we have

$$
N(T)=M(T)+S(T)
$$

where

$$
\begin{aligned}
M(T) & =\frac{-1}{\pi} \int_{0}^{T} \operatorname{Re} \frac{\zeta^{\prime}}{\zeta^{\prime}}\left(\frac{1}{2}+i t\right) d t \\
S(T) & =\frac{1}{\pi} \int_{\infty}^{1 / 2} \operatorname{Im} \frac{\zeta^{\prime}}{\zeta}(\sigma+i T) d \sigma=\frac{1}{\pi} \arg \zeta\left(\frac{1}{2}+i T\right) .
\end{aligned}
$$

The argument is defined by continuous horizontal movement from $\infty+i T$ to $\frac{1}{2}+i T$ starting with the value zero. Meanwhile, comparing (2.3) with the zero counting formula shown in Davenport [1], we see that

$$
\begin{aligned}
M(t)= & \frac{1}{\pi}\left[\arg \left(-\frac{1}{2}+i t\right)+\arg \left(\pi^{-(1 / 2)(1 / 2+i t)}\right)\right. \\
& \left.+\arg \Gamma\left(\frac{5}{4}+i \frac{t}{2}\right)\right] \\
= & \frac{t}{2 \pi}\left(\log \frac{t}{2 \pi}-1\right)+O(1) \quad \text { for large } t
\end{aligned}
$$


and

$$
M^{\prime}(t) \sim \frac{1}{2 \pi} \log t
$$

Our first object is to construct the following "explicit formula."

Proposition 1. For $\alpha \geq 0$, we have

$$
R_{\alpha}(u)=\int_{0}^{\infty} \frac{\sin _{\alpha}(t u)}{t^{\alpha+1}} d S(t)
$$

Before proceeding with the proof of Proposition 1, we need several lemmas.

LEMMA 2.1. If $\alpha \geq 0, u>0$, and $f(v)=e^{s v}$, then

$$
\left(I_{\alpha} f\right)(u)=\frac{1}{\Gamma(\alpha)} \int_{0}^{u}(u-v)^{\alpha-1} e^{s v} d v=\frac{e^{s u}}{s^{\alpha}}+L(\alpha, s, u)
$$

for $s$ on the slit plane cut along the line from the origin to $-i \infty$; and $s^{\alpha}$ is defined by analytic continuation starting with $1^{\alpha}=1$. Moreover, $\left(I_{\alpha} f\right)(u)$ is a holomorphic function on the entire plane of $s$ with $L(\alpha, s, u) \ll_{\alpha} u^{\alpha-1} /|s|+\left(|\sigma u|^{\alpha-1}+1\right) /|s|^{\alpha}, \sigma=\operatorname{Re} s$, and we define $L(0, s, u) \equiv 0$.

Proof. We start by considering, for $\alpha>0$,

$$
\begin{aligned}
e^{-s u} & \frac{1}{\Gamma(\alpha)} \int_{0}^{u}(u-v)^{\alpha-1} e^{s v} d v=\frac{1}{\Gamma(\alpha)} \int_{0}^{u}(u-v)^{\alpha-1} e^{s(v-u)} d v \\
& =\frac{1}{s^{\alpha}} \frac{1}{\Gamma(\alpha)} \int_{0}^{s u} v^{\alpha-1} e^{-v} d v \\
& =\frac{1}{s^{\alpha}} \frac{1}{\Gamma(\alpha)}\left\{\Gamma(\alpha)-\int_{s u}^{\sigma u} v^{\alpha-1} e^{-v} d v-\int_{\sigma u}^{\infty} v^{\alpha-1} e^{-v} d v\right\} \\
& =\frac{1}{s^{\alpha}}+O_{\alpha}\left(\frac{|s u|^{\alpha-1}}{|s|^{\alpha}} e^{-\sigma u}+\frac{|\sigma u|^{\alpha-1}+1}{|s|^{\alpha}} e^{-\sigma u}\right) ;
\end{aligned}
$$

the estimate of this remainder term will be given at the end of this section. This proves Lemma 2.1 by taking, for $\alpha>0$,

$$
L(\alpha, s, u)=-\frac{1}{s^{\alpha}} \frac{e^{s u}}{\Gamma(\alpha)}\left\{\int_{s u}^{\sigma u} v^{\alpha-1} e^{-v} d v+\int_{\sigma u}^{\infty} v^{\alpha-1} e^{-v} d v\right\} .
$$

REMARK. We see by Lemma 2.1 that

$$
\begin{gathered}
\frac{1}{\Gamma(\alpha)} \int_{0}^{u}(u-v)^{\alpha-1} \sinh s v d v=\left(\sinh _{\alpha} s\right)(u) \\
\quad=\frac{1}{2}\left[\frac{e^{s u}}{s^{\alpha}}+\frac{e^{-s u}}{(-s)^{\alpha}}\right]+\widetilde{L}(\alpha, s, u)
\end{gathered}
$$


where $\widetilde{L}(\alpha, s, u)$ is bounded by

$$
O\left(\frac{u^{\alpha-1}}{|s|}+\frac{|\sigma u|^{\alpha-1}+1}{|s|^{\alpha}}\right)
$$

and $\widetilde{L}(0, s, u) \equiv 0$.

LEMMA 2.2. Consider, for $\alpha \geq 0$ and $u>0,\left(\sinh _{\alpha} s\right)(u)$ as defined by (2.7) on the slit plane in Lemma 2.1. Then for $c>0, \alpha \geq 0, \eta \geq 0$

$$
\begin{aligned}
& \lim _{T \rightarrow \infty} \frac{1}{\pi i} \int_{c-i T}^{c+i T} \frac{\left(\sinh _{\alpha} s\right)(u)}{s} e^{-s \eta} d s \\
& = \begin{cases}\frac{1}{\Gamma(\alpha+1)}(u-\eta)^{\alpha} & (u>\eta), \\
0 & (u<\eta) \text { or }(u=\eta, \alpha>0), \\
\frac{1}{2} & (u=\eta, \alpha=0) .\end{cases}
\end{aligned}
$$

Proof. The result follows by (1.5.3) of Titchmarsh [7] and (2.7).

Proof of Proposition 1. Consider, for $c>1$ and $T>0$ not an ordinate of a zero of $\zeta(s)$,

$$
J(T)=\frac{1}{2 \pi i} \int_{c-i T}^{c+i T} \frac{\left(\sinh _{\alpha}\left(s-\frac{1}{2}\right)\right)(u)}{s-\frac{1}{2}} \frac{\zeta^{\prime}}{\zeta}(s) d s ;
$$

note that $\left(\sinh _{\alpha} s\right)(u) / s$ is an even function of $s$.

Since $\frac{\zeta^{\prime}}{\zeta}(s) \ll(\log |t|)^{2}$ for $s=\sigma+i t\left(-\frac{1}{2} \leq \sigma\right)$ and a suitable sequence of $t$ with infinity the limit of $|t|$, we have that by computation of residues and for large $T$

$$
\begin{aligned}
J(T)= & -\frac{\sinh _{\alpha}\left(\frac{1}{2} u\right)}{\left(\frac{1}{2}\right)^{\alpha+1}}-\int_{0}^{T} \frac{\left(\sin _{\alpha} t\right)(u)}{t} d M(t) \\
& +\sum_{\substack{0<\gamma<T \\
\zeta(1 / 2+i \gamma)=0}} \frac{\sin _{\alpha}(\gamma u)}{\gamma^{\alpha+1}}+o(1) \\
= & -\frac{\sinh _{\alpha}\left(\frac{1}{2} u\right)}{\left(\frac{1}{2}\right)^{\alpha+1}}+\int_{0}^{T} \frac{\sin _{\alpha}(t u)}{t^{\alpha+1}} d S(t)+o(1),
\end{aligned}
$$

since $\left(\sin _{\alpha} t\right)(u) / t=\sin _{\alpha}(t u) / t^{\alpha+1}$. On the other hand, by using

$$
\frac{\zeta^{\prime}}{\zeta}(s)=-\sum_{n=1}^{\infty} \Lambda(n) n^{-1 / 2} e^{-(s-1 / 2) \log n},
$$


we have

$$
J(T)=-\sum_{n=1}^{\infty} \Lambda(n) n^{-1 / 2} \frac{1}{2 \pi i} \int_{c-i T}^{c+i T} \frac{\left(\sinh _{\alpha}\left(s-\frac{1}{2}\right)\right)(u)}{s-\frac{1}{2}} e^{-(s-1 / 2) \log n} d s .
$$

By virtue of Lemma 2.2, (2.2), and making $T \rightarrow \infty$, we get

$$
R_{\alpha}(u)=\int_{0}^{\infty} \frac{\sin _{\alpha}(t u)}{t^{\alpha+1}} d S(t)
$$

This completes the proof of Proposition 1.

We now give the estimate of the remainder term in the proof of Lemma 2.1. It is based on the following

Proposition 2. If $s=\sigma+i$ is on the slit plane cut along the line from the origin to $-i \infty$, then, for $\alpha>0$,

$$
\int_{\sigma+i t}^{\infty+i t} v^{\alpha-1} e^{-v} d v=O_{\alpha}\left(e^{-\sigma}\left(|s|^{\alpha-1}+1\right)\right)
$$

with $t \geq 0$, or $t \leq 0$ and $\sigma>0$, and

$$
\int_{\sigma+i t}^{-\infty+i t} v^{\alpha-1} e^{v} d v=O_{\alpha}\left(e^{-\sigma}\left(|s|^{\alpha-1}+1\right)\right)
$$

with $t \geq 0$, or $t \leq 0$ and $\sigma<0$.

Proof. We only prove the first assertion, and the second assertion will follow by a similar argument.

If $-1 \leq \sigma \leq 1$, then, for $|t|<1$,

$$
\int_{\sigma+i t}^{\infty+i t} v^{\alpha-1} e^{-v} d v=O(1)=O\left(e^{-\sigma}\right),
$$

and for $|t|>1$, by integrating by parts $[\alpha]$ times,

$$
\begin{aligned}
\int_{\sigma+i t}^{\infty+i t} v^{\alpha-1} e^{-v} d v & =-\left.e^{-v} v^{\alpha-1}\right|_{v=\sigma+i t} ^{v=\infty+i t}+(\alpha-1) \int_{\sigma+i t}^{\infty+i t} v^{\alpha-2} e^{-v} d v \\
& =O\left(e^{-\sigma}|s|^{\alpha-1}\right)+O\left(\int_{\sigma+i t}^{\infty+i t}|v|^{\alpha-[\alpha]-2} e^{-v} d v\right) \\
& =O\left(e^{-\sigma}|s|^{\alpha-1}\right)+O\left(e^{-\sigma} \int_{\sigma+i t}^{\infty+i t}|v|^{\alpha-[\alpha]-2} d v\right) \\
& =O\left(e^{-\sigma}|s|^{\alpha-1}\right) .
\end{aligned}
$$


So, for $-1 \leq \sigma \leq 1$, we have

$$
\int_{\sigma+i t}^{\infty+i t} v^{\alpha-1} e^{-v} d v=O\left(e^{-\sigma}\left(|s|^{\alpha-1}+1\right)\right)
$$

If $1<\sigma$, then $|s|>1$ and

$$
\begin{aligned}
\int_{\sigma+i t}^{\infty+i t} v^{\alpha-1} e^{-v} d v & =-\left.e^{-v} v^{\alpha-1}\right|_{v=\sigma+i t} ^{v=\infty+i t}+(\alpha-1) \int_{\sigma+i t}^{\infty+i t} v^{\alpha-2} e^{-v} d v \\
& =O\left(e^{-\sigma}|s|^{\alpha-1}\right)+O\left(\int_{\sigma+i t}^{\infty+i t}|v|^{\alpha-[\alpha]-2} e^{-v} d v\right) \\
& =O\left(e^{-\sigma}|s|^{\alpha-1}\right)+O\left(e^{-\sigma} \int_{\sigma+i t}^{\infty+i t}|v|^{\alpha-[\alpha]-2} d v\right) \\
& =O\left(e^{-\sigma}|s|^{\alpha-1}\right) .
\end{aligned}
$$

Finally if $\sigma<-1$, then $|s|>1$ and

$$
\begin{aligned}
\int_{\sigma+i t}^{\infty+i t} v^{\alpha-1} e^{-v} d v= & \int_{\sigma+i t}^{-1+i t} v^{\alpha-1} e^{-v} d v+\int_{-1+i t}^{\infty+i t} v^{\alpha-1} e^{-v} d v \\
= & -\left.e^{-v} v^{\alpha-1}\right|_{v=\sigma+i t} ^{v=-1+i t}+(\alpha-1) \int_{\sigma+i t}^{-1+i t} v^{\alpha-2} e^{-v} d v \\
& +O\left(e^{-\sigma}\left(|1+i t|^{\alpha-1}+1\right)\right), \quad \text { by }(2.8) \\
= & O\left(e^{-\sigma}\left(|s|^{\alpha-1}+|1+i t|^{\alpha-1}\right)\right) \\
& +O\left(e^{-\sigma} \int_{\sigma+i t}^{-1+i t}|v|^{\alpha-2} d v\right) \\
& +O\left(e^{-\sigma}\left(|1+i t|^{\alpha-1}+1\right)\right) \\
= & O\left(e^{-\sigma}\left(|s|^{\alpha-1}+|1+i t|^{\alpha-1}+1\right)\right) \\
= & O\left(e^{-\sigma}\left(|s|^{\alpha-1}+1\right)\right)
\end{aligned}
$$

This completes the proof of the first assertion of Proposition 2.

Now we obtain from Proposition 2

$$
\int_{\sigma u}^{\infty} v^{\alpha-1} e^{-v} d v=O\left(e^{-\sigma u}\left(|\sigma u|^{\alpha-1}+1\right)\right)
$$

needed in the proof of Lemma 2.1.

As for the following quantity

$$
\int_{s u}^{\sigma u} v^{\alpha-1} e^{-v} d v
$$


we apply the Cauchy integral theorem and get for $\sigma>0$, or $\sigma<0$ and $t>0$,

$$
\begin{aligned}
\int_{s u}^{\sigma u} v^{\alpha-1} e^{-v} d v & =\int_{s u}^{\infty+i t u} v^{\alpha-1} e^{-v} d v+\int_{\infty}^{\sigma u} v^{\alpha-1} e^{-v} d v \\
& =O\left(e^{-\sigma u}\left(|s u|^{\alpha-1}+|\sigma u|^{\alpha-1}+1\right)\right),
\end{aligned}
$$

by Proposition 2; and if $t<0$ and $\sigma<0$, then by the Cauchy integral theorem

$$
\begin{aligned}
\int_{s u}^{\sigma u} v^{\alpha-1} e^{-v} d v & =(-1)^{\alpha-1} \int_{-\sigma u}^{-s u} v^{\alpha-1} e^{v} d v \\
& =(-1)^{\alpha-1}\left\{\int_{-\infty}^{-\sigma u} v^{\alpha-1} e^{v} d v+\int_{-s u}^{-\infty+i t u} v^{\alpha-1} e^{v} d v\right\} \\
& =O\left(e^{-\sigma u}\left(|s u|^{\alpha-1}+|\sigma u|^{\alpha-1}+1\right)\right)
\end{aligned}
$$

by the second assertion of Proposition 2.

Hence we have given the estimates in the proof of Lemma 2.1.

3. Theorems. Consider equation $(\Delta)$ in Proposition 1. On taking integration by parts $m+1$ times, we get formally

$$
\sqrt{\frac{2}{\pi}} \frac{R_{\alpha}(u)}{u^{m+1}}=(-1)^{m+1} \int_{0}^{\infty} \frac{S_{m}^{*}(t)}{t^{\alpha+1}} K(\alpha, m, t u) d t
$$

where

$$
K(\alpha, m, \theta)=\sqrt{\frac{2}{\pi}} \theta^{\alpha+1}\left(\frac{d}{d \theta}\right)^{m+1} \frac{\sin _{\alpha}(\theta)}{\theta^{\alpha+1}} \quad(\theta>0) .
$$

Since for $\alpha \geq 0$, by considering the Taylor series expansion of $\sin \theta$,

$$
\sin _{\alpha} \theta=\sum_{n=0}^{\infty} \frac{(-1)^{n}}{\Gamma(2 n+2+\alpha)} \theta^{2 n+1+\alpha} \quad(\theta>0),
$$

and the series on the right-hand side is defined for any real $\alpha \in \mathbb{R}$, we define (3.2) for any real $\alpha \in \mathbb{R}$.

In this section, we give suitable conditions on real $\alpha$ and integer $m$ for which $K(\alpha, m, \theta)$ defines a bounded and invertible transform on $L^{2}(0, \infty)$. In view of $S(t)=O(\log t / \log \log t),(0.5)$, and (0.6); we see that

$$
\frac{S_{m}^{*}(t)}{t^{\alpha+1}} \in L^{2}(0, \infty)
$$

for $\frac{1}{2}<\alpha-m+1<\frac{3}{2}, m \geq 0, \alpha \geq 0$. 
We show first

THEOREM 1. Equation (3.1) holds for $\alpha>m-1, \alpha \geq 0, m=$ $0,1,2,3, \ldots$.

The following lemma is helpful in the proof of Theorem 1.

LEMMA 3.1. For a function $f(x)$ on $[0, \infty)$, put

$$
M(\alpha, x)=\sup _{0 \leq t \leq x}\left|f_{\alpha}(t)\right|
$$

Then the following estimate holds uniformly in $\alpha$ :

$$
\left|f_{\alpha}(t)\right| \ll M^{1-\alpha}(0, t) \cdot M^{\alpha}(1, t) \quad(0 \leq \alpha \leq 1) .
$$

Proof. Note first that if either $g(x)$ or $h(x)$ is monotonic on $[a, b]$, then

$$
\int_{a}^{b} g(x) d h(x) \ll \sup _{a \leq x \leq b}|g(x)| \cdot \sup _{a \leq x \leq b}|h(x)| .
$$

We may suppose $f(x)$ is non-constant.

Now for $0<\lambda<x$

$$
\begin{aligned}
f_{\alpha}(x)= & \frac{1}{\Gamma(\alpha)} \int_{0}^{x}(x-y)^{\alpha-1} f(y) d y \\
= & \frac{1}{\Gamma(\alpha)} \int_{0}^{x-\lambda}(x-y)^{\alpha-1} f(y) d y \\
& +\frac{1}{\Gamma(\alpha)} \int_{x-\lambda}^{x}(x-y)^{\alpha-1} f(y) d y \\
= & \frac{1}{\Gamma(\alpha)} \int_{0}^{x-\lambda}(x-y)^{\alpha-1} d f_{1}(y) \\
& -\frac{1}{\Gamma(\alpha+1)} \int_{x-\lambda}^{x} f(y) d(x-y)^{\alpha} \\
\ll & \alpha \lambda^{\alpha-1} M(1, x)+\lambda^{\alpha} M(0, x) \\
\ll & M^{1-\alpha}(0, x) M^{\alpha}(1, x),
\end{aligned}
$$

by taking $\lambda=\alpha M(1, x) / M(0, x)<x$.

This proves Lemma 3.1.

Proof of Theorem 1. It suffices to show that the integral constant

$$
\left.S_{m}^{*}(t)\left(\frac{d}{d t}\right)^{m} \frac{\sin _{\alpha}(t u)}{t^{\alpha+1}}\right|_{t=0} ^{t=\infty}=0
$$


for $\alpha>m-1, \alpha \geq 0, m \geq 0$. Lemma 3.1 and the power series (3.3) give

$$
\sin _{\beta}(\theta) \ll \begin{cases}\max \left(\theta^{\beta-1}, 1\right), & \theta \rightarrow+\infty \\ \theta^{\beta+1}, & \theta \rightarrow 0^{+}\end{cases}
$$

for all $\beta \in \mathbb{R}$. Thus, the Leibniz formula yields

$$
\begin{aligned}
\left(\frac{d}{d t}\right)^{m} \frac{\sin _{\alpha}(t u)}{t^{\alpha+1}} & \ll \sum_{j=0}^{m}\left(\begin{array}{c}
m \\
j
\end{array}\right)\left|\sin _{\alpha-j}(t u)\right| \cdot t^{-\alpha-1-m+j} \\
& \ll \max \left\{t^{-m-2}, t^{-\alpha-1}\right\} \quad(t \rightarrow+\infty),
\end{aligned}
$$

and the power series (3.3) gives

$$
\left(\frac{d}{d t}\right)^{m} \frac{\sin _{\alpha}(t u)}{t^{\alpha+1}} \ll 1 \quad\left(t \rightarrow 0^{+}\right) .
$$

Now (3.5) follows immediately from (0.5), (0.6), and (3.7), (3.8). This proves Lewmma 3.2 Theorem 1.

We next show the following.

TheOREM 2. The kernel (3.2) defines a bounded and invertible transform on $L^{2}(0, \infty)$ for $m-\frac{1}{2}<\alpha<m+\frac{3}{2}, m=-1,0,1,2, \ldots$ In particular, for $\alpha=m=-1,0,1,2, \ldots$, it defines an involution on $L^{2}(0, \infty)$.

The proof of Theorem 2 is based on the following lemma.

LEMMA 3.2. We have

$$
K(\alpha+1, m+1, \theta)=\left(\mathrm{Id}-(m+\alpha+3) A_{m+2}\right) K(\alpha, m, \theta)
$$

for $m+\alpha+3>0,-\infty<\alpha<+\infty, m=-1,0,1,2,3, \ldots$

Proof. Recall (3.2) and (3.3). We see that

$$
K(\alpha, m, \theta)=\sqrt{\frac{2}{\pi}} \theta^{\alpha+1}\left(\frac{d}{d \theta}\right)^{m+1} \sum_{l=0}^{\infty} \frac{(-1)^{l}}{\Gamma(2 l+\alpha+2)} \theta^{2 l} .
$$


Thus

$$
\begin{aligned}
(m+\alpha & +3) A_{m+2} K(\alpha, m, \theta) \\
= & \sqrt{\frac{2}{\pi}}(m+\alpha+3) \theta^{-m-2} \int_{0}^{\theta} \eta^{m+1} \eta^{\alpha+1}\left(\frac{d}{d \eta}\right)^{m+1} \\
& \cdot \sum_{l=0}^{\infty} \frac{(-1)^{l}}{\Gamma(2 l+\alpha+2)} \eta^{2 l} d \eta \\
= & \sqrt{\frac{2}{\pi}} \theta^{-m-2}\left\{\left.\eta^{m+\alpha+3}\left(\frac{d}{d \eta}\right)^{m+1} \sum_{l=0}^{\infty} \frac{(-1)^{l}}{\Gamma(2 l+\alpha+2)} \eta^{2 l}\right|_{\eta=0} ^{\eta=\theta}\right. \\
= & \left.K(\alpha, m, \theta) \int_{0}^{\theta} \eta^{m+\alpha+3}\left(\frac{d}{d \eta}\right)^{m+2} \sum_{l=0}^{\infty} \frac{(-1)^{l}}{\Gamma(2 l+\alpha+2)} \eta^{2 l} d \eta\right\} \\
& -\sqrt{\frac{2}{\pi}} \theta^{-m-2} \int_{0}^{\theta} \eta^{m+\alpha+3}\left(\frac{d}{d \eta}\right)^{m+2} \sum_{l=0}^{\infty} \frac{(-1)^{l}}{\Gamma(2 l+\alpha+2)} \eta^{2 l} d \eta \\
= & K(\alpha, m, \theta)-K(\alpha+1, m+1, \theta) .
\end{aligned}
$$

This proves Lemma 3.2.

Proof of Theorem 2. Note that $K(\alpha,-1, \theta)=\sqrt{\frac{2}{\pi}} \sin _{\alpha}(\theta)$ and $K(-1,-1, \theta)=\sqrt{\frac{2}{\pi}} \cos \theta$. Lemma 3 and Theorem 1 of Kueh [5] show that $K(\alpha,-1, \theta)\left(-\frac{3}{2}<\alpha<\frac{1}{2}\right)$ defines a bounded and invertible transform on $L^{2}(0, \infty)$. Thus Theorem 2 follows immediately from Lemma 3.2 and Lemmas 1.1, 1.2, 1.3, 1.4. We see also that the kernel $K^{-1}(\alpha, m, \theta)$ of the inverse of the transform defined by $K(\alpha, m, \theta)$ satisfies, for the same condition as in Lemma 3.2,

$$
K^{-1}(\alpha+1, m+1, \theta)=\left(\mathrm{Id}-(m+\alpha+3) A_{\alpha+2}\right) K^{-1}(\alpha, m, \theta)
$$

and

$$
K^{-1}(\alpha,-1, \theta)=\sqrt{\frac{2}{\pi}} \sin \left(\theta-\frac{\pi}{2}\right) \quad\left(-\frac{3}{2}<\alpha<\frac{1}{2}\right) .
$$

By Theorems 1, 2, and (3.4), equation (0.8) holds.

Finally, we prove the following

THEOREM 3. Equation (0.7) holds for $\alpha>m-1, \alpha \geq 0, m=$ $0,1,2, \ldots$. 
We need the estimate

$$
R_{1}(u)=-\frac{\pi}{2} M^{\prime}(0) u+O(1)
$$

in the proof of Theorem 3. Taking $\alpha=1$ in Proposition 1, we obtain

$$
R_{1}(u)=\int_{0}^{\infty} \frac{1-\cos t u}{t^{2}} d S(t) \text {. }
$$

Note that $S(t)=N(t)-M(t)$. So

$$
\begin{aligned}
R_{1}(u)= & \int_{0}^{\infty} \frac{1-\cos t u}{t^{2}} d N(t)-\int_{0}^{\infty} \frac{1-\cos t u}{t^{2}} M^{\prime}(t) d t \\
= & -M^{\prime}(0) \int_{0}^{\infty} \frac{1-\cos t u}{t^{2}} d t \\
& -\int_{0}^{\infty} \frac{1-\cos t u}{t^{2}}\left(M^{\prime}(t)-M^{\prime}(0)\right) d t+O(1) \\
= & -\frac{\pi}{2} M^{\prime}(0) u+O(1),
\end{aligned}
$$

since

$$
\int_{0}^{\infty} \frac{1-\cos t u}{t^{2}} d t=\frac{\pi}{2} u
$$

and $M^{\prime}(t)$ is an even function making $M^{\prime}(t)-M^{\prime}(0)=O\left(t^{2}\right)$ as $t \rightarrow$ $0^{+}$, and $M^{\prime}(t) \sim \frac{1}{2 \pi} \log t$ for large $t$, by $(2.6)$.

Proof of Theorem 3. By Theorem 2,

$$
\frac{S_{1}^{*}(t)}{t^{2}}=\sqrt{\frac{2}{\pi}} \lim _{U \rightarrow \infty} \int_{0}^{U} \frac{R_{1}(u)}{u^{2}} K(1,1, t u) d u .
$$

Now estimate (3.10) makes the above integral converge in the ordinary sense. Thus

$$
S_{1}^{*}(t)=\frac{2}{\pi} \int_{0}^{\infty} R_{1}(u)\left(\frac{d}{d u}\right)^{2} \frac{\sin _{1}(t u)}{u^{2}} d u .
$$

In addition,

$$
\int_{0}^{U} R_{1}(u)\left(\frac{d}{d u}\right)^{2} \frac{\sin _{1}(t u)}{u^{2}} d u
$$

is bounded uniformly with respect to $t$ in any compact set as $U \rightarrow \infty$ So, after applying fractional integral operator on both sides of (3.11), we get for $\alpha \geq 0$

$$
S_{\alpha+1}^{*}(t)=\frac{2}{\pi} \int_{0}^{\infty} R_{1}(u)\left(\frac{d}{d u}\right)^{2} \frac{\sin _{\alpha+1}(t u)}{u^{\alpha+2}} d u
$$


Now set

$$
f(t)=\frac{2}{\pi} \int_{0}^{\infty} R_{1}(u)\left(\frac{d}{d u}\right)^{2} \frac{\sin _{\alpha}(t u)}{u^{\alpha+1}} d u \quad(\alpha>0) .
$$

By using Lemma 2 of Kueh [5] and (3.6), we see that similarly for $\alpha>0$

$$
\int_{0}^{U} R_{1}(u)\left(\frac{d}{d u}\right)^{2} \frac{\sin _{\alpha}(t u)}{u^{\alpha+1}} d u
$$

is bounded uniformly with respect to $t$ in any compact set as $U \rightarrow \infty$. Hence, on integrating both sides of (3.13), we get

$$
f_{1}(t)=\frac{2}{\pi} \int_{0}^{\infty} R_{1}(u)\left(\frac{d}{d u}\right)^{2} \frac{\sin _{\alpha+1}(t u)}{u^{\alpha+2}} d u \quad(\alpha>0)
$$

and, in view of (3.12),

$$
S_{\alpha+1}^{*}(t)=f_{1}(t) \quad(\alpha>0) .
$$

Thus, on differentiating both sides of (3.14), we get

$$
\begin{aligned}
S_{\alpha}^{*}(t) & =f(t)=\frac{2}{\pi} \int_{0}^{\infty} R_{1}(u)\left(\frac{d}{d u}\right)^{2} \frac{\sin _{\alpha}(t u)}{u^{\alpha+1}} d u \\
& =-\frac{2}{\pi} \int_{0}^{\infty} R(u) \frac{d}{d u} \frac{\sin _{\alpha}(t u)}{u^{\alpha+1}} d u \quad(\alpha>0) .
\end{aligned}
$$

We now repeat the same argument as in Theorem 1 and get

$$
\frac{S_{\alpha}^{*}(t)}{t^{m+1}}=(-1)^{m+1} \sqrt{\frac{2}{\pi}} \int_{0}^{\infty} \frac{R_{m}(u)}{u^{\alpha+1}} K(\alpha, m, t u) d u
$$

with $\alpha>m-1, \alpha>0, m=0,1,2, \ldots$. Also by $(0.2)$, the above equation holds for $\alpha=m=0$.

This completes the proof of Theorem 3.

The author takes pleasure in thanking Professor P. X. Gallagher for his suggestions and many helpful discussions on the subject.

\section{REFERENCES}

[1] H. Davenport, Multiplicative Number Theory, Springer-Verlag, second edition, 1980.

[2] P. X. Gallagher, Applications of Guinand's explicit formula, Proc. 1984 Stillwater Conference on Analytic Number Theory and Diophantine Problems, BaselStuttgart-Boston, 1987.

[3] A. Guinand, A summation formula in the theory of prime numbers, Proc. London Math. Soc., (2) 50 (1948), 107-119. 
[4] G. H. Hardy, J. E. Littlewood and G. Polya, Inequalities, Cambridge University Press, 1951.

[5] K.-L. Kueh, Interpolated Fourier transforms, Real Analysis Exchange, 14(2) (1988-89), 321-344.

[6] J. E. Littlewood, On the zeros of the Riemann zeta function, Proc. Camb. Phil. Soc., 22 (1924), 295-318.

[7] E. C. Titchmarsh, Introduction to the Theory of Fourier Integrals, Oxford University Press, 1948.

Received January 23, 1989 and in revised form October 25, 1990.

INSTITUTE OF MATHEMATICS

ACADEMIa Sinica

Nankang, TaIPEI, TAIwan 11529 


\section{PACIFIC JOURNAL OF MATHEMATICS EDITORS}

\author{
V. S. VARADARAJAN \\ (Managing Editor) \\ University of California \\ Los Angeles, CA 90024-1555-05 \\ Herbert Clemens \\ University of Utah \\ Salt Lake City, UT 84112 \\ THOMAS ENRIGHT \\ University of California, San Diego \\ La Jolla, CA 92093
}

R. FINN

Stanford University

Stanford, CA 94305

HeRmann FlaschKa

University of Arizona

Tucson, AZ 85721

VAUGHAN F. R. JoNES

University of California

Berkeley, CA 94720

SteVen KeRCKHOFF

Stanford University

Stanford, CA 94305
C. C. MOORE

University of California

Berkeley, CA 94720

MaRTin ScharLemanN

University of California

Santa Barbara, CA 93106

HAROLd STARK

University of California, San Diego

La Jolla, CA 92093

\section{ASSOCIATE EDITORS \\ R. ARENS \\ E. F. BECKENBACH (1906-1982) \\ B. H. NeumanN \\ F. WoLf \\ (1904-1989) \\ K. YoshidA \\ SUPPORTING INSTITUTIONS \\ UNIVERSITY OF ARIZONA \\ UNIVERSITY OF BRITISH COLUMBIA \\ CALIFORNIA INSTITUTE OF TECHNOLOGY \\ UNIVERSITY OF CALIFORNIA \\ MONTANA STATE UNIVERSITY \\ UNIVERSITY OF NEVADA, RENO \\ NEW MEXICO STATE UNIVERSITY OREGON STATE UNIVERSITY \\ UNIVERSITY OF OREGON \\ UNIVERSITY OF SOUTHERN CALIFORNIA \\ STANFORD UNIVERSITY \\ UNIVERSITY OF HAWAII \\ UNIVERSITY OF TOKYO \\ UNIVERSITY OF UTAH \\ WASHINGTON STATE UNIVERSITY UNIVERSITY OF WASHINGTON}

The Supporting Institutions listed above contribute to the cost of publication of this Journal, but they are not owners or publishers and have no responsibility for its content or policies.

Mathematical papers intended for publication in the Pacific Journal of Mathematics should be in typed form or offset-reproduced (not dittoed), double spaced with large margins. Please do not use built up fractions in the text of the manuscript. However, you may use them in the displayed equations. Underline Greek letters in red, German in green, and script in blue. The first paragraph must be capable of being used separately as a synopsis of the entire paper. In particular it should contain no bibliographic references. Please propose a heading for the odd numbered pages of less than 35 characters. Manuscripts, in triplicate, may be sent to any one of the editors. Please classify according to the 1991 Mathematics Subject Classification scheme which can be found in the December index volumes of Mathematical Reviews. Supply name and address of author to whom proofs should be sent. All other communications should be addressed to the managing editor, or Elaine Barth, University of California, Los Angeles, California 90024-1555-05.

There are page-charges associated with articles appearing in the Pacific Journal of Mathematics. These charges are expected to be paid by the author's University, Government Agency or Company. If the author or authors do not have access to such Institutional support these charges are waived. Single authors will receive 50 free reprints; joint authors will receive a total of 100 free reprints. Additional copies may be obtained at cost in multiples of 50 .

The Pacific Journal of Mathematics (ISSN 0030-8730) is published monthly except for July and August. Regular subscription rate: $\$ 190.00$ a year (10 issues). Special rate: $\$ 95.00$ a year to individual members of supporting institutions.

Subscriptions, orders for numbers issued in the last three calendar years, and changes of address should be sent to Pacific Journal of Mathematics, P.O. Box 969, Carmel Valley, CA 93924, U.S.A. Old back numbers obtainable from Kraus Periodicals Co., Route 100, Millwood, NY 10546.

The Pacific Journal of Mathematics at P.O. Box 969, Carmel Valley, CA 93924 (ISSN 0030-8730) is published monthly except for July and August. Second-class postage paid at Carmel Valley, California 93924, and additional mailing offices. Postmaster: send address changes to Pacific Journal of Mathematics, P.O. Box 969, Carmel Valley, CA 93924.

PUBLISHED BY PACIFIC JOURNAL OF MATHEMATICS, A NON-PROFIT CORPORATION Copyright (C) 1991 by Pacific Journal of Mathematics 


\section{Pacific Journal of Mathematics}

Vol. 150, No. $2 \quad$ October, 1991

Selman Akbulut and Henry Churchill King, Rational structures on

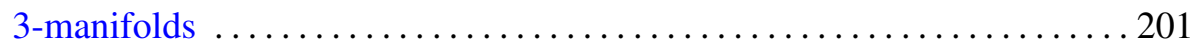

Mark Baker, On coverings of figure eight knot surgeries $\ldots \ldots \ldots \ldots \ldots 215$

Christopher Michael Brislawn, Traceable integral kernels on countably generated measure spaces . ...........................229

William Chin, Crossed products and generalized inner actions of Hopf algebras

Tadeusz Figiel, William Buhmann Johnson and Gideon Schechtman, Factorizations of natural embeddings of $l_{p}^{n}$ into $L_{r}$. II $\ldots \ldots \ldots \ldots \ldots 261$

David Howard Gluck, Character value estimates for groups of Lie type ... 279

Charn-Huen Kan, Norming vectors of linear operators between $L_{p}$

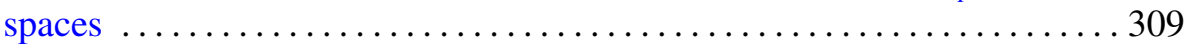

Marko Kranjc, Embedding a 2-complex $K$ in $\mathbb{R}^{4}$ when $H^{2}(K)$ is a cyclic

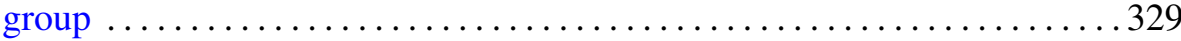

Ka-Lam Kueh, The remainder terms aspect of the theory of the Riemann

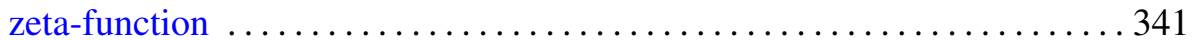

J. A. Marti, Sur la rigidité comparée de fonctions, distributions, ou hyperfonctions analytiques par rapport à un groupe de variables

Margherita Roggero and Paolo Valabrega, Chern classes and cohomology for rank 2 reflexive sheaves on $\mathbf{P}^{3}$ 